\title{
Enema avoids colonoscopy rescheduling
}

Published online: 30 May 2012

(C) Springer Healthcare 2012

MedWire News: Enema can rescue colonoscopy in patients with inadequately prepared bowels, suggests research published in Colorectal Disease.

"The enema prevents the inconvenience of rescheduling with its increased costs, possible loss of earnings and continued anxiety regarding the possibility of colonic pathology," say Akira Horiuchi (Showa Inan General Hospital, Komagane, Japan) and co-authors.

The team investigated the use of enema for bowel preparation in 504 patients referred for afternoon colonoscopy with propofol sedation, and given polyethylene glycol (PEG) solution the morning before the procedure.

Patients with poor or inadequate bowel preparation at colonoscopy were then given a $500 \mathrm{~mL}$ PEG enema instilled at the level of the hepatic flexure. The patients were allowed to recover from sedation, and colonoscopy was attempted again after bowel evacuation.

Poor or inadequate bowel preparation was detected at colonoscopy in 26 (4.9\%) of the patients. This was attributed to inability or unwillingness to take 1 L of PEG $(n=4)$, or incomplete bowel evacuation after consumption of 2 L PEG $(n=22)$.

The patients spent an average of 52.5 minutes in the lavatory after enema, "suggesting that establishing a minimum time before reintroducing the colonoscope such as 45 or 50 minutes might be useful," say Horiuchi et al.

Colonoscopy was successful in all 25 patients who achieved good or excellent bowel preparation, and in the remaining patient with fair bowel preparation. There were no complications, and one patient was diagnosed with invasive colorectal cancer, and seven with adenoma.

One-day bowel preparation was taken at 09.00, and all the enema patients left hospital by 18.00 compared with $\mathbf{1 6 . 0 0}$ for patients with successful colonoscopy on first attempt.

The team concludes: "A rescue enema avoided the need for postponement of colonoscopy in patients with a poor bowel preparation after a one-day PEG bowel preparation regimen."

By Lynda Williams

\section{Reference}

Colorectal Dis 2012; Advance online publication 